\title{
SEM Evaluation of the plant-pollinator interactions between nitidulid beetles and a native tropical Malvaceae species, Gossypium tomentosum on Kauai.
}

\author{
K. N. Burraston, J. S. Gardner, and G. M. Booth \\ Dept of Integrative Biology, 401 WIDB, Brigham Young University, Provo, UT 84602.
}

On Kauai, HI Gossypium tomentosum was observed being heavily visited by a nitidulid beetle. Gossypium tomentosum is a member of the Malvaceae family and the only member of the cotton genus endemic to the Hawaiian Islands (1). The beetle is a nitidulid, Aethina concolor, commonly known as a "sap beetle". Sap beetles are found world- wide and are attracted to plants with sweet nectar or juices from decaying fruit (2). There are many reasons a beetle may visit a flower. First, they may be foraging for food such as nectar or pollen, second, they may be using the flower as a mating site, and thirdly, they may be seeking refuge from external temperatures (3).

The purpose of this study was to determine the reason for the abundance of the beetles present on the Gossypium flora and how the structure of the beetle, Aethina concolor, related to the pollination of the plant Gossypium tomentosum. Examining the location of the pollen on the beetle, the structure of the beetle, the contents of the beetle gut, and the various types and structure of the pollen on the beetle tested the preliminary hypothesis that the presence of the beetle was due to its consumption of the Gossypium pollen.

Nitidulid beetles were collected from Gossypium plants at the National Tropical Botanical Gardens located on Kauai. One set of collections was preserved in $70 \%$ ethanol. The other set of beetles was collected and stored dry in tissue paper to keep pollen undisturbed on the beetle body. ESEM analysis was conducted using a $10 \mathrm{KV}$ accelerating potential at a working distance of $10 \mathrm{~mm}$ and at a low vacuum to reduce charging problems with the specimens.

Pollen was concentrated on the head and mouth regions.(Fig 1) The abundance of pollen around the mandibles supports the hypothesis that the nitidulids are foraging for pollen on the Gossypium flowers. Pollen found in the mandibles of the nitidulids confirms that the size of the nitidulid jaws is compatible with the size of the Gossypium pollen. (Fig 2) The pollen found in the dissected gut of the nitidulids confirms the pollen as part of the beetle diet. (Fig 3) Only Gossypium pollen was found on the beetles collected. This is evidence that nitidulids have limited foraging areas and a preference for the Gossypium sp. The morphology of the pollen is typical of the Malvaceae family (1). The burr like structure of the pollen is adapted for clinging to pollinators as a method of dispersal. This is the mechanism that makes the nitidulids a pollination vector for the Gossypium sp. (Fig 4)

Beetles and other invertebrate pollinators are critically important in the pollination of plants, and yet, our knowledge of the interactions between these insect pollinators and the plants they visit is far from thoroughly documented (3). This SEM study shows evidence that nitidulid beetles are an important pollinator for Malvaceae species and begins to explain the mechanisms and limitations of nitidulid beetle pollination. Identifying the pollination biology specifics of this plant will benefit in the managing of its populations. On a broader scale, the experiment will contribute to the knowledge regarding the role nitidulids play in plant pollination. Further study is needed to document the role of nitidulid beetles in Gossypium pollination. 


\section{References}

1. D.R. Dejoode, and J. Wendal. American Journal of Botany 79 (1992) 1311.

2. D. H. Habeck, American Beetles Volume 2: Nitidulidae. CRC Press: New York, 2002.

3. P. Bemhardt, Plant Systematics and Evolution 222 (2000) 293.
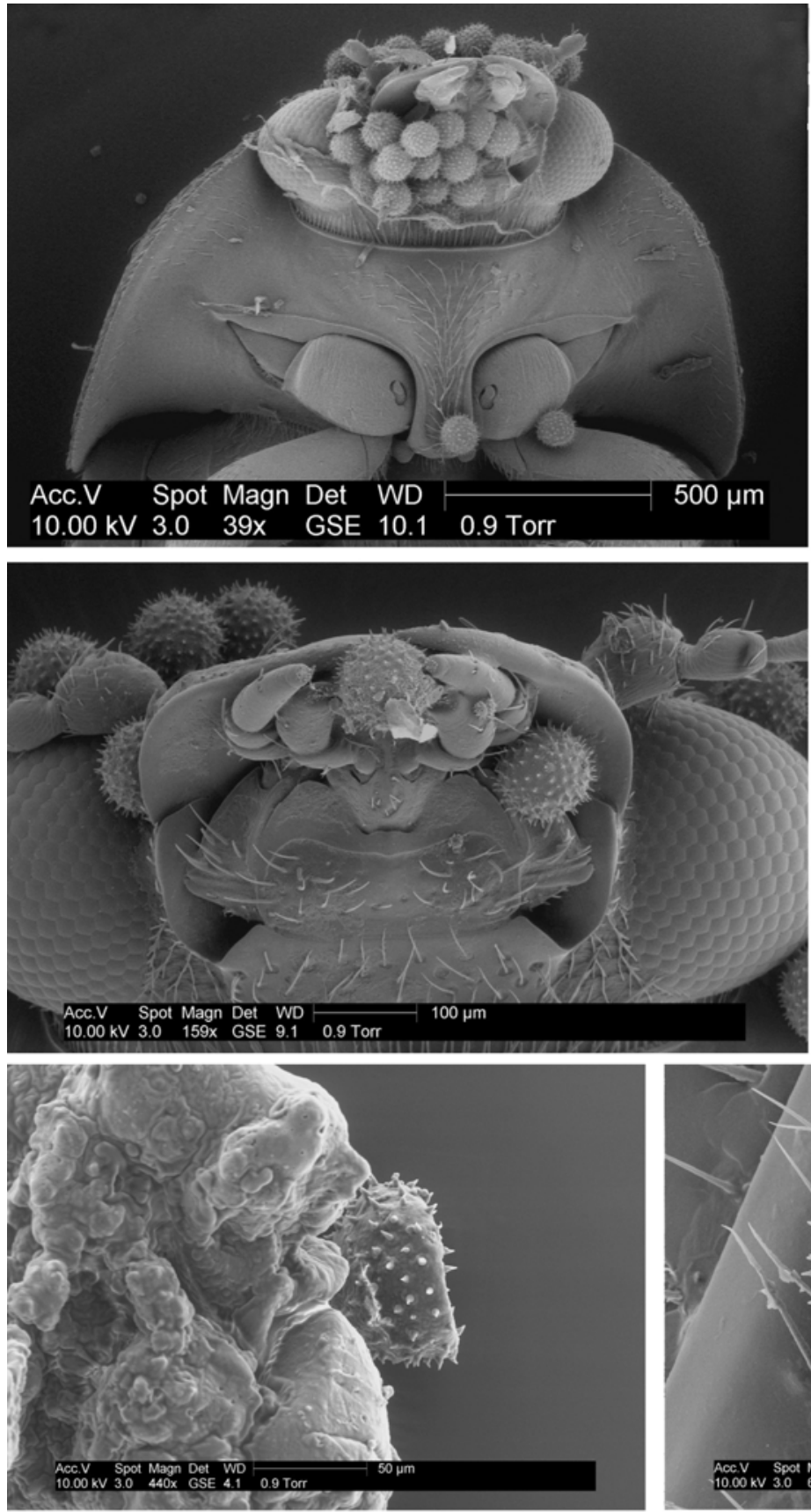

Fig. 3 - Gossypium pollen and gut tissue dissected from nitidulid beetle.

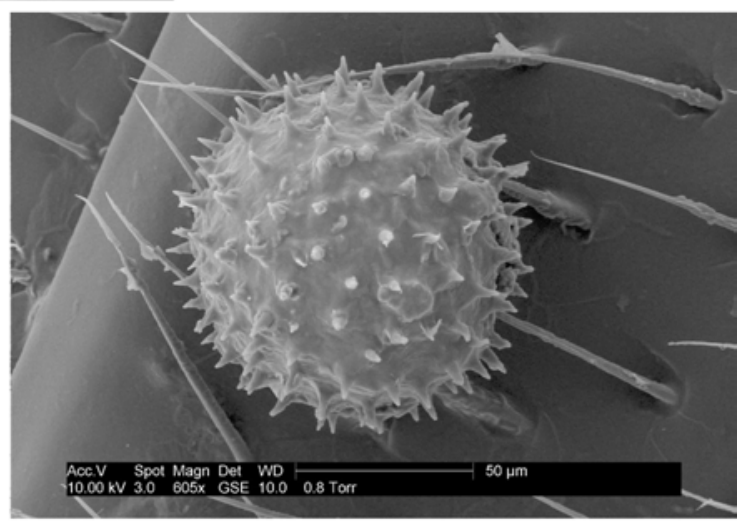

Fig. 4 - Malvaceae pollen grain entangled in nitidulid beetle hairs. 